\title{
Preliminary Spectral Analysis of the NLS1 Galaxy WPVS48
}

\author{
M.A.Probst and W. Kollatschny \\ Institut für Astrophysik, Göttingen University \\ Friedrich-Hund-Platz 1 \\ 37073 Göttingen \\ Germany
}

Received: August 15, 2019; Accepted: October 3, 2019

\begin{abstract}
A spectroscopic campaign was carried out on the Narrow Line Seyfert 1 (NLS1) Galaxy WPVS 48 (2MASX J09594263-3112581) at the Southern African Large Telescope (SALT) from December 2013 to June 2014. The final objective is to perform reverberation mapping on the acquired light curves in order to calculate the Black Hole (BH) mass and to characterise the Broad Line Region (BLR) of the galaxy. To date, the spectra of one third of the observations are reduced and intercalibrated. Therefore, we will provide spectra displaying the spectral variability of WPVS 48 and preliminary light curves of the continuum at $\lambda 5100 \AA$ as well as the emission lines $\mathrm{H} \alpha, \mathrm{H} \beta$ and $\mathrm{HeII}$ $\lambda 4686$.
\end{abstract}

Key words: galaxies: active - galaxies: NLS1 - galaxies: nuclei - galaxies: individual: WPVS 48

\section{Introduction}

Among Active Galactic Nuclei (AGN), the subclass of NLS1 galaxies is identified by the widths of their optical Balmer lines (FWHM $\leq 2000 \mathrm{~km} \mathrm{~s}^{-1}$ ) as well as a relatively weak $[\mathrm{OIII}] \lambda 5007$ emission in comparison to $\mathrm{H} \beta$ (Osterbrock \& Pogge, 1985). Little is known about whether the variability pattern of NLS1 differs from those of Broad Line Seyfert 1 Galaxies (BLS1). The method of reverberation mapping, which is used to study the internal structure of AGN as well as their BH mass, is very time consuming and was therefore applied to less than a handful of NLS1s (Pozo Nuñez et al., 2014; Pei et al., 2014; Huang et al., 2019, e.g.). We will use light curves comprising of data obtained in various nights over the course of several months in order to calculate the mass of the $\mathrm{BH}$ and to study the BLR structure.

WPVS 48 is a NLS1 galaxy at the distance of $161 \mathrm{Mpc}$ with a redshift of $z=0.0377$ (Véron-Cetty \& Véron, 2010). Its host is a spiral galaxy and has a luminous nucleus $(V=14.78)$, as it was shown by Winkler (1997). Photometric reverberation mapping on WPVS 48 was realised by Pozo Nuñez et al. (2014) using data in the B, R, J and $\mathrm{K}$ bands. The retrieved light curves from all these 
bands display high variability and the found sharp dust echo argues for a faceon torus. In the subsequent discussion, Pozo Nuñez et al. (2014) proposed two scenarios:

1. A flattened inner region of the torus coming close to the accretion disk in the equatorial plane.

2. The torus being geometrically and optically thick and only the facing rim being visible to the observer.

For our investigation, spectroscopic SALT data is used. In our analysis we consider the continuum at $\lambda 5100 \AA$ and the emission lines $\mathrm{H} \alpha, \mathrm{H} \beta$ and HeII $\lambda 4686$. The analysis of the spectrum will be extended in the near future.

\section{Observations and Data Reduction}

We obtained 27 spectra with the SALT $10 \mathrm{~m}$ telescope over the time period from December 1, 2013 to June 29, 2014. The Robert Stobie Spectrograph with a PG0900 grating was used at all nights of observation. The observations are taken at the same airmass as a result of the construction of the telescope. The slit width was fixed to 2 arcsecs.

The optical spectra were taken in the wavelength regime from 4350 to $7375 \AA$, this corresponds to the wavelengths of 4200 to $7100 \AA$ in the rest frame. Spectra taken at SALT are divided into 3 segments. Hence, two gaps are seen, the first being between 5363 and $5415 \AA$ ( 5162 and $5226 \AA$ in rest frame) and the second one between 6408 and $6454 \AA$ (6172 and $6227 \AA$ in rest frame).

Flat field, arc images (xenon and argon lamps) and spectra of standard stars were taken for the reduction and calibration process. The spectra were reduced in a homogeneous way (bias subtraction, cosmic ray correction, flat-field correction, 2D-wavelength calibration, night-sky subtraction and flux calibration) using IRAF reduction packages according to (Kollatschny et al., 2018). No further denoising was performed.

To date, the spectra of 9 out of 27 nights of observation were reduced, covering the time period from December 1, 2013 to February 7, 2014. LTT 4364 was used as the standard star for the flux calibration. Following data reduction and flux calibration, the spectra were intercalibrated according to the fluxes of the narrow $[\mathrm{OIII}]$ and $[\mathrm{SII}]$ lines, as these are considered to remain constant over timescales of years. Constant, narrow lines should vanish in the rms spectrum, when the spectra are intercalibrated. This was tested including further narrow $[\mathrm{OI}]$ and $[\mathrm{NII}]$ lines.

\section{Results}

We present three reduced and intercalibrated spectra of WPVS 48 in Fig. 1. The spectra were obtained on December 13, 2013, December 26, 2013 and February 
7, 2014. These spectra show variability in both continuum and line fluxes. All wavelengths are given in the rest frame.

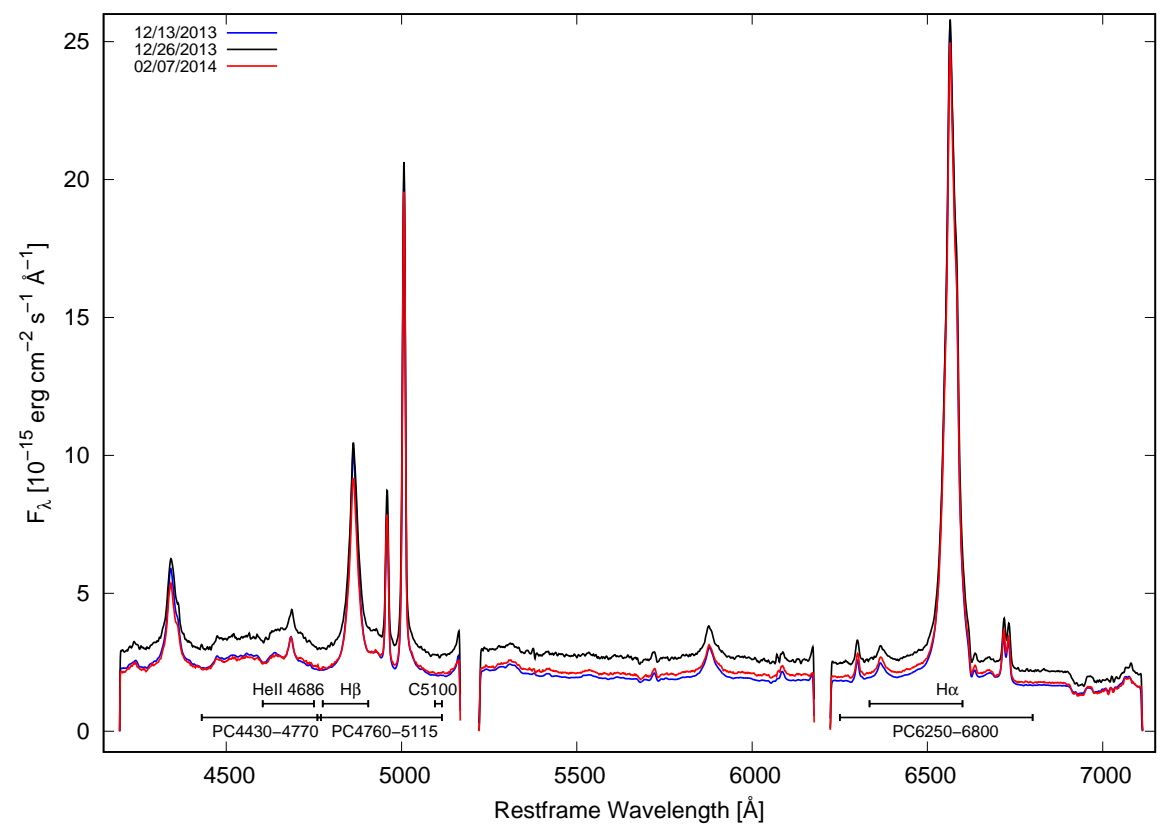

Figure 1. Optical spectra of WPVS 48 taken with the SALT telescope for our variability campaign on December 13, 2013, December 26, 2013 and February 7, 2014.

The mean flux density of the continuum is usually measured at the wavelength of $5100 \AA$, as this wavelength regime is free of strong emission and absorption lines. Furthermore, three sections of the spectra were chosen to create pseudo-continua for each spectrum. These sections encompass the wavelengths between $6250 \AA$ and $6800 \AA, 4760 \AA$ and $5115 \AA$ as well as $4430 \AA$ and $4770 \AA$ respectively for $\mathrm{H} \alpha, \mathrm{H} \beta$ and HeII $\lambda$ 4686. These pseudo-continua are also displayed in Fig. 1.

We integrated the flux densities of the two Balmer lines $\mathrm{H} \alpha$ and $\mathrm{H} \beta$ as well as the helium line HeII $\lambda$ 4686. The integration limits are given in Tab. 1 and included into Fig. 1. Before integration, the pseudo-continua were substracted from the corresponding spectrum. The resulting, preliminary light curves are presented in Fig. 2. The preliminary light curves cover the period from December 1, 2013 till February 7, 2014. In this time, the variability in the emission lines are measured to be $19 \%$ for $\mathrm{H} \alpha, 17 \%$ for $\mathrm{H} \beta$ and $66 \%$ for HeII $\lambda 4686$. For the continuum at $\lambda 5100$, we found a variability of $31 \%$. For comparison, Pozo Nuñez 


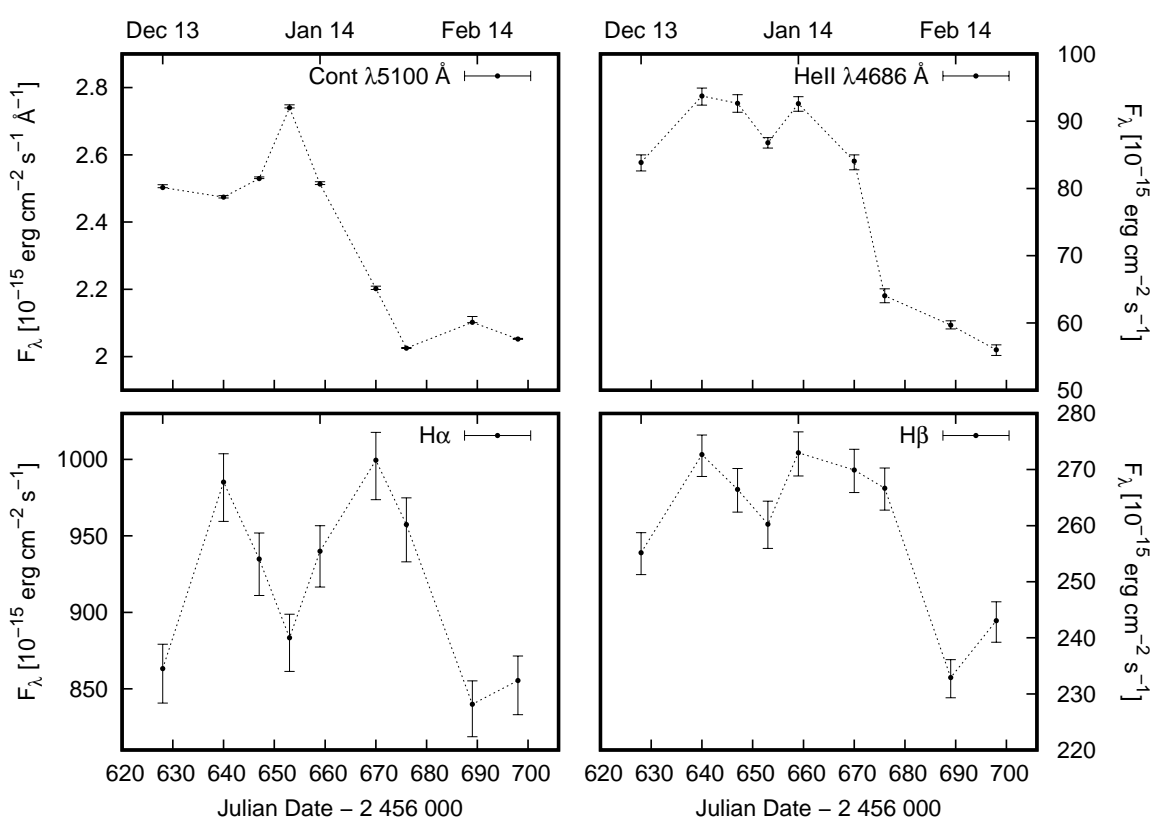

Figure 2. Preliminary light curves of the continuum flux density at $5100 \AA$ (in units of $10^{-15} \mathrm{erg} \mathrm{cm}^{-1} \AA^{-1}$ ) as well as integrated line fluxes of $\mathrm{H} \alpha, \mathrm{H} \beta$ and $\mathrm{HeI} \lambda 5876$ (in units of $10^{-15} \mathrm{erg} \mathrm{cm}^{-1}$ ) for our variability campaign between December 2013 and February 2014.

Table 1. Rest frame continuum boundaries and integration limits.

\begin{tabular}{lcc}
\hline $\begin{array}{l}\text { Cont./Line } \\
(1)\end{array}$ & $\begin{array}{c}\text { Wavelength range } \\
(2)\end{array}$ & $\begin{array}{c}\text { Pseudo-continuum } \\
(3)\end{array}$ \\
\hline Cont. 5100 & $5095 \AA-5115 \AA$ & \\
H $\alpha$ & $6335 \AA-6600 \AA$ & $6250 \AA-6800 \AA$ \\
$\mathrm{H} \beta$ & $4775 \AA-4905 \AA$ & $4760 \AA-5115 \AA$ \\
HeII $\lambda 4686$ & $4604 \AA-4750 \AA$ & $4430 \AA-4770 \AA$ \\
\hline \hline
\end{tabular}

et al. (2014) found a variability of roughly $13 \%$ in both the B and R band, which encompass the $\mathrm{H} \beta$ and $\mathrm{H} \alpha$ line, respectively. 

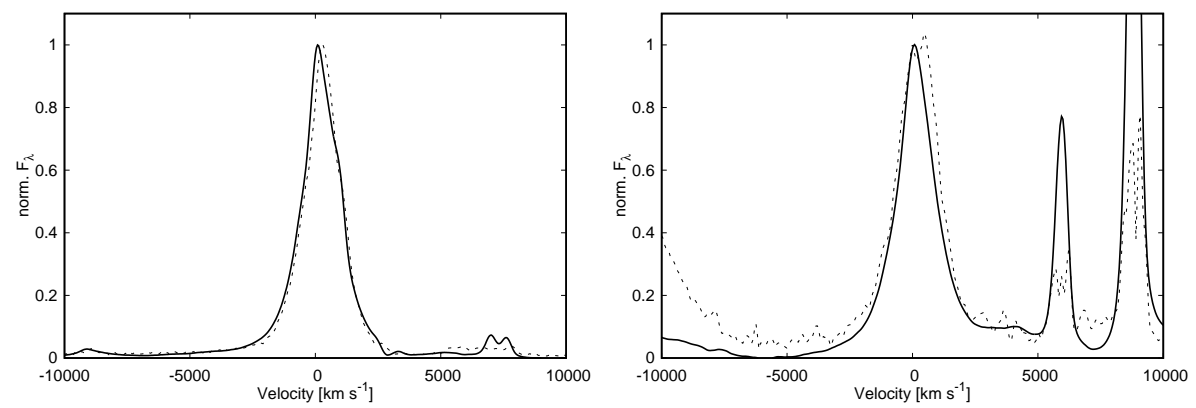

Figure 3. Normalised mean (solid) and rms (dashed) line profile of $\mathrm{H} \alpha$ (a) and $\mathrm{H} \beta$ (b) in velocity space.

The mean line profiles of $\mathrm{H} \alpha$ and $\mathrm{H} \beta$ in the velocity space are presented in Fig 3. Both the mean and rms profiles are determined in reference to the available data of the 9 nights of observation and after the pseudo-continua were subtracted. The mean profiles show both narrow and broad components. The FWHM was calculated for both lines based on the rms spectrum. As a result, we obtained $1890 \pm 60 \mathrm{~km} \mathrm{~s}^{-1}$ for $\mathrm{H} \beta$ and $1660 \pm 50 \mathrm{~km} \mathrm{~s}^{-1}$ for $\mathrm{H} \alpha$.

\section{Summary and Outlook}

The spectra and light curves reveal flux variations of WPVS 48 in the continuum as well as in Balmer and He emission lines. The number of reduced nights is yet not sufficient to perform a robust cross correlation and hence, neither the mass of the BH nor any details about the BLR are presented. Agreeing with the results of Pozo Nuñez et al. (2014), we observed high variability in the emission lines. We also confirm that the FWHM of $\mathrm{H} \beta$ corresponds to a galaxy of type NLS1.

Acknowledgements. This work has been supported by DFG grant Ko 857/33-1. We also want to thank Martin W. Ochmann for his helpful advice.

\section{References}

Huang, Y.-K., Hu, C., Zhao, Y.-L., et al., Reverberation Mapping of the Narrow-line Seyfert 1 Galaxy I Zwicky 1: Black Hole Mass. 2019, Astron. J., 876, 14

Kollatschny, W., Ochmann, M., Zetzl, M., et al., Broad-line region structure and line profile variations in the changing look AGN HE 1136-2304. 2018, Astron. Astrophys., 619, A168

Osterbrock, D. \& Pogge, R., The Spectra of Narrow-Line Seyfert 1 Galaxies. 1985, Astrophys. J., 297, 166 
Pei, L., Barth, A. J., Aldering, G. S., et al., Reverberation Mapping of The Keplerfield AGN KA1858+4850. 2014, Astron. J., 795, 15

Pozo Nuñez, F., Haas, M., Chini, R., et al., Dust reverberation-mapping of the Seyfert 1 galaxy WPVS48. 2014, Astron. Astrophys., 561, L8

Véron-Cetty, M.-P. \& Véron, P., A catalogue of quasars and active nuclei: 13th edition. 2010, Astron. Astrophys., 518, A10

Winkler, H., The extinction, flux distribution and luminosity of Seyfert 1 nuclei derived from $\operatorname{UBV}(\mathrm{Rl})_{\mathrm{C}}$ aperture photometry. 1997, Mon. Not. R. Astron. Soc., 292, 273 